\title{
Compatibility fans realizing graphical nested complexes
}

\author{
Thibault Manneville $\|$ and Vincent Pilaud非 \\ ${ }^{1}$ LIX, École Polytechnique, France \\ ${ }^{2}$ CNRS \& LIX, École Polytechnique, France
}

\begin{abstract}
Abstact. Graph associahedra are polytopes realizing the nested complex $\mathcal{N}(\mathrm{G})$ on connected subgraphs of a graph $\mathrm{G}$. While all known explicit constructions produce polytopes with the same normal fan, the great variety of fan realizations of classical associahedra and the analogy between finite type cluster complexes and nested complexes incited us to transpose S. Fomin and A. Zelevinsky's construction of compatibility fans for generalized associahedra (2003) to graph associahedra. Using a compatibility degree, we construct one fan realization of $\mathcal{N}(\mathrm{G})$ for each of its facets. Specifying G to paths and cycles, we recover a construction by F. Santos for classical associahedra (2011) and extend F. Chapoton, S. Fomin and A. Zelevinsky's construction (2002) for type $B$ and $C$ generalized associahedra.

Résumé. Les associaèdres de graphe sont des réalisations polytopales du complexe emboîté $\mathcal{N}(\mathrm{G})$ des sous-graphes connexes d'un graphe G. Si toutes les constructions explicites connues produisent des polytopes avec le même éventail normal, la pléthore de réalisations de l'associaèdre classique et l'analogie entre complexes amassés de type fini et complexes emboîtés nous ont incités à transposer la construction des associaèdres généralisés comme éventails de compatibilité par S. Fomin et A. Zelewinsky (2003) aux associaèdres de graphe. Grâce à un degré de compatibilité, nous construisons un éventail simplicial réalisant $\mathcal{N}(\mathrm{G})$ pour chacune de ses facettes. Quand $\mathrm{G}$ est un chemin ou un cycle, nous retrouvons une construction de F. Santos de l'associaèdre classique (2011) et étendons celle de F. Chapoton, S. Fomin et A. Zelewinsky (2002) pour les associaèdres généralisés de type $B$ et $C$.
\end{abstract}

Keywords. Graph associahedra, finite type cluster algebras, compatibility degrees, compatibility fans

Many associahedra. The $n$-dimensional associahedron is a simple polytope whose vertices correspond to Catalan objects (triangulations, binary trees, ...) and whose edges correspond to mutations between them (diagonal flips, edge rotations, ...). It was first realized as a convex polytope by M. Haiman (unpublished) and C. Lee [Lee89]. Since then many other polytopal constructions were found. J.-L. Loday [Lod04] gave a combinatorial construction based on binary trees, later extended by C. Hohlweg and C. Lange [HL07] to produce $\Theta\left(2^{n}\right)$ geometrically nonequivalent realizations of the $n$-dimensional associahedron (meaning that their normal fans are not linearly equivalent). Another combinatorial construction by F. Santos, published in a paper of C. Ceballos, F. Santos and G. Ziegler [CSZ15, Section 5], produces asymptotically

\footnotetext{
${ }^{\ddagger}$ Email: manneville@lix.polytechnique.fr. Supported by an École Polytechnique Gaspard Monge doctoral grant.

$\S$ Email: pilaudelix.polytechnique.fr. Partially supported by the French ANR grants EGOS (12 JS02 002 01) and SC3A (15 CE40 000401$)$.
}

1365-8050 @ 2016 Discrete Mathematics and Theoretical Computer Science (DMTCS), Nancy, France 

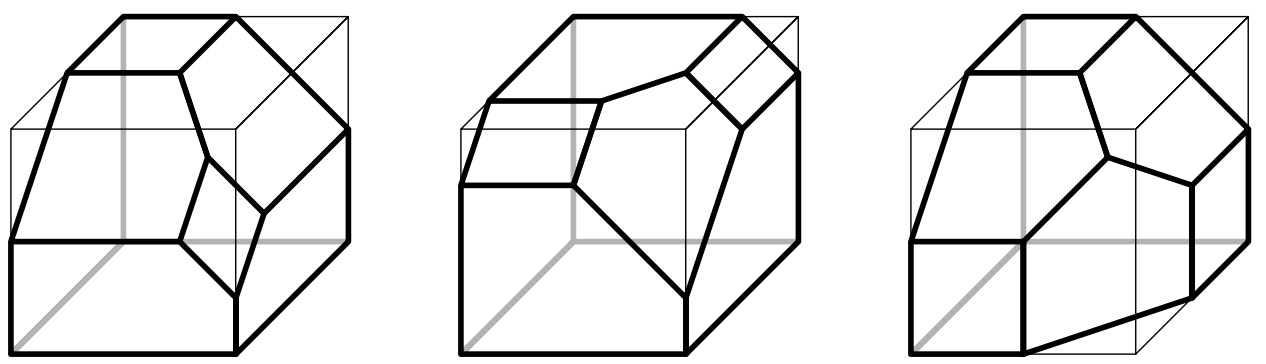

Fig. 1: All nonequivalent realizations of the 3-dimensional associahedra by F. Santos, including that of F. Chapoton, S. Fomin and A. Zelevinsky (left). Figure from [CSZ15], with permission.

Catalan many geometrically nonequivalent constructions (see Figure 1). Both families intersect only in one realization, due to F. Chapoton, S. Fomin and A. Zelevinsky [CFZ02].

This extended abstract reports on our paper [MP15] on two generalizations of classical associahedra, namely graph associahedra realizing graphical nested complexes [CD06] and generalized associahedra realizing finite cluster complexes [FZ02, FZ03]. We extend F. Santos' construction to obtain many simplicial fan realizations for graphical nested complexes. As byproducts, we get new constructions of type $B$ and $C$ generalized associahedra, including that of F. Chapoton, S. Fomin and A. Zelevinsky [CFZ02].

Graph associahedra. Given a simple graph G, the G-associahedron Asso $(G)$ is a simple polytope whose polar has a face lattice isomorphic to the nested complex Nest $(\mathrm{G})$ on $\mathrm{G}$. The latter is the simplicial complex whose vertices, called tubes, are connected induced subgraphs of $\mathrm{G}$, and whose facets, called tubings, are sets of pairwise compatible (i.e. nested or disjoint and nonadjacent) tubes. Figure 2 shows that the graph associahedra of certain special families of graphs coincide with well-known families of polytopes. Graph associahedra were realized as convex polytopes by successive truncations of faces of a simplex [CD06], as Minkowski sums of faces of a simplex [Pos09, [FS05], or from their normal fans by exhibiting explicit inequality descriptions [Zel06]. For a given graph $\mathrm{G}$, the resulting polytopes all have the same normal fan which coarsens the type $A$ Coxeter arrangement. Alternative realizations with different normal fans were obtained by successive truncations of a cube in [Vol10, DFRS15]. Yet the resulting normal fans are not explicitly described, nor compared. We provide a new unrelated family of complete simplicial fans realizing the nested complex Nest $(G)$ for any graph $G$.

Cluster algebras and cluster fans. Our construction is directly inspired from properties of finite type cluster algebras introduced by S. Fomin and A. Zelevinsky in [FZ02, FZ03]. It follows the connection between nested complexes and cluster complexes already pointed out by A. Zelevinsky in [Zel06].

A cluster algebra $\mathcal{A}$ is a commutative algebra generated by cluster variables grouped into clusters, obtained from an initial cluster by successive mutations. A mutation exchanges a single variable in a cluster according to a polynomial equality [FZ02]. Two cluster variables are compatible if they belong to a same cluster and exchangeable if they are exchanged in a mutation. The cluster complex of $\mathcal{A}$ is the simplicial complex on cluster variables of $\mathcal{A}$ whose facets are the clusters of $\mathcal{A}$. Finite type cluster algebras (i.e. with a finite cluster complex) are classified by crystallographic root systems [FZ03].

The Laurent Phenomenon [FZ02] asserts that each cluster variable $x$ can be expressed as a Laurent polynomial in terms of the cluster variables $x_{1}^{\circ}, \ldots, x_{n}^{\circ}$ of the initial cluster $X^{\circ}$. The d-vector of $x$ with respect to $X^{\circ}$ is the vector $\mathbf{d}\left(X^{\circ}, x\right)$ whose $i$ th coordinate is the exponent of the initial variable $x_{i}^{\circ}$ 

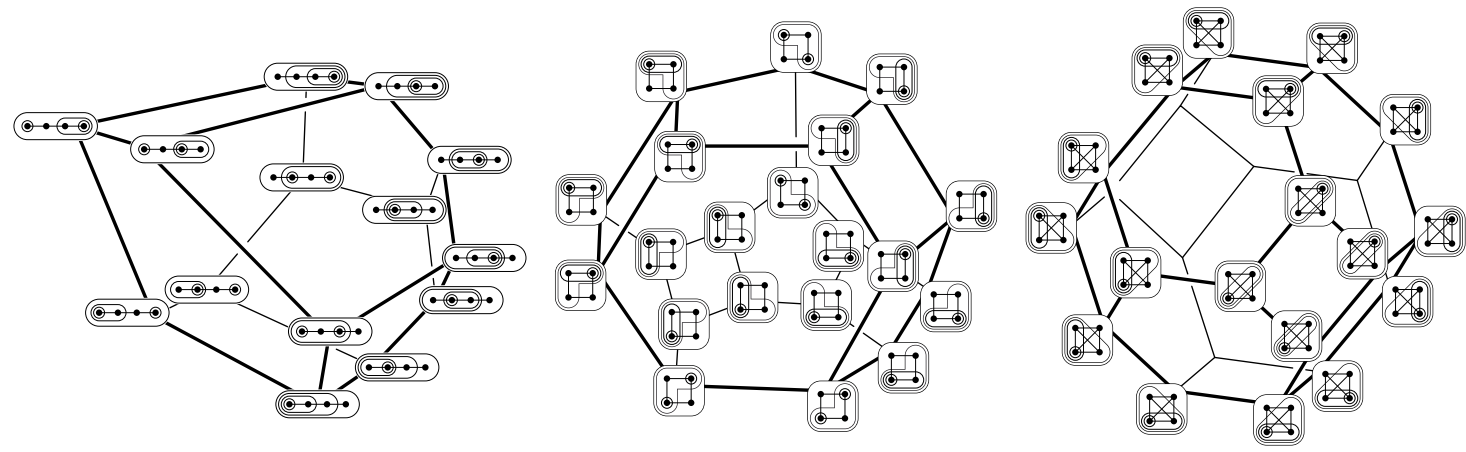

Fig. 2: Some classical polytopes are graph associahedra: the classical associahedron is the path associahedron (left), the cyclohedron is the cycle associahedron (right) and the permutahedron is the complete graph associahedron.

in the denominator of $x$. In finite type, this value was interpreted in [FZ03, CP15] as the compatibility degree $\left(x_{i}^{\circ} \| x\right)$ between the cluster variables $x_{i}^{\circ}$ and $x$. It only depends on $x_{i}^{\circ}$ and $x$ and has the following properties: for any distinct cluster variables $x$ and $x^{\prime}$, we have $\left(x \| x^{\prime}\right) \geq 0$ with equality if and only if $x$ and $x^{\prime}$ are compatible, and $\left(x \| x^{\prime}\right)=1=\left(x^{\prime} \| x\right)$ if and only if $x$ and $x^{\prime}$ are exchangeable. The d-vectors can be used to construct a simplicial fan realization of the cluster complex, called d-vector fan: for certain initial clusters in finite type cluster algebras, the cones generated by the $\mathbf{d}$-vectors of all collections of compatible cluster variables form a complete simplicial fan realizing the cluster complex. S. Fomin and A. Zelevinsky [FZ03] show it for the bipartite initial cluster, S. Stella [Ste13] for all acyclic initial clusters, and F. Santos [CSZ15, Section 5] for any initial cluster in type $A$. We expect this property to hold for any initial cluster of any finite type cluster algebra. A consequence of our results is the following statement.

Theorem 1 Let $\mathcal{A}$ be any cluster algebra of finite type $A, B$ or $C$. For any initial cluster $X^{\circ}$ of $\mathcal{A}$, the $\mathbf{d}$-vectors with respect to $X^{\circ}$ support a complete simplicial fan realizing the cluster complex of $\mathcal{A}$.

This statement is a corollary of our main result which concerns all graph associahedra (see Theorem 3 . In all papers mentioned above, the polytopality was also shown for all constructed fans. Even if we could not show polytopality for all graph associahedra, we however prove it for finite cluster complexes.

Theorem 2 All fans constructed in Theorem[1 are polytopal. More precisely, any $\mathbf{d}$-vector fan in type A, B or $C$ is the normal fan of a simple polytope, realizing the corresponding generalized associahedron.

Overview. Our construction is an analogue of the d-vector fan for any graph associahedron Asso $(G)$. We define an asymmetric notion of compatibility degree on the tubes of $\mathrm{G}$, with similar properties as the compatibility degree in cluster complexes. Given an initial maximal tubing $\mathrm{T}^{\circ}$ on $\mathrm{G}$ and a tube $\mathrm{t}$ of $\mathrm{G}$, we use our compatibility degree to define the primal (resp. dual) compatibility vector of $\mathrm{t}$ with respect to $T^{\circ}$. Duality arises from the degree's asymmetry. We will often abbreviate "primal compatibility" to "compatibility".

Although no denominator is involved anymore, we keep the letter $\mathbf{d}$ for compatibility degree vector, and to match with the cluster algebra notations. Indeed, our compatibility degrees coincide with these of [FZ03] in types $A, B$, and $C$ : compatibility degrees on type $A$ cluster variables correspond to compatibility (and dual compatibility) degrees on tubes of paths while compatibility degrees on type $C$ (resp. $B$ ) cluster variables correspond to compatibility (resp. dual compatibility) degrees on tubes of cycles. 
In Section 1, we define precisely the previous notions on graph associahedra and state our main result.

Theorem 3 For any graph $\mathrm{G}$, the primal (resp. dual) compatibility vectors of the tubes of $\mathrm{G}$ with respect to any initial maximal tubing on $\mathrm{G}$ support a complete simplicial fan realizing the nested complex $\mathcal{N}(\mathrm{G})$.

In Section 2, we detail how type $A, B$ and $C$ cluster complexes arise as path and cycle associahedra and thus how Theorem 3 implies Theorem 1 . We moreover explain there how we derive Theorem 2. We sketch in Section 3 the proof of Theorem 3 . We finally discuss in Section 4 the number of different realizations we get, with graph-analog results of F. Santos' Catalan many realizations [CSZ15, Section 5].

\section{Graph associahedra and compatibility fans}

Fix a graph $\mathrm{G}$ with vertex set $\mathrm{V}$, let $\kappa(\mathrm{G})$ be its set of connected components, and set $n:=|\mathrm{V}|-|\kappa(\mathrm{G})|$. A tube of $G$ is a nonempty subset $t$ of vertices of $G$ inducing a connected subgraph $G[t]$ of $G$. The inclusion maximal tubes of $\mathrm{G}$ induce its connected components $\kappa(\mathrm{G})$; all other tubes are called proper. Two tubes $\mathrm{t}, \mathrm{t}^{\prime}$ of $\mathrm{G}$ are compatible if they are either nested $\left(\mathrm{t} \subseteq \mathrm{t}^{\prime}\right.$ or $\left.\mathrm{t}^{\prime} \subseteq \mathrm{t}\right)$, or disjoint and nonadjacent $\left(t \cup t^{\prime}\right.$ is not a tube of $G$ ). A tubing on $G$ is a set $T$ of pairwise compatible proper tubes of $G$. The collection of all tubings on $\mathrm{G}$ is a simplicial complex, called nested complex of $\mathrm{G}$ and denoted by $\mathcal{N}(\mathrm{G})$.

For a tubing $T$ on $G$ and a tube $t$ of $T \cup \kappa(G)$, we define $\lambda(t, T):=t \backslash \bigcup_{\mathrm{t}^{\prime} \in \mathrm{T}, \mathrm{t}^{\prime} \subseteq \mathrm{t}} \mathrm{t}^{\prime}$. It follows from the definitions that the sets $\lambda(t, T)$ for $t \in T \cup \kappa(G)$ form a partition of the vertex set of $G$. When moreover $T$ is maximal, one can see that each set $\lambda(\mathrm{t}, \mathrm{T})$ contains a unique vertex of $\mathrm{G}$, that we call the root of $\mathrm{t}$ in $\mathrm{T}$.

We call flip a pair of distinct maximal tubings $\mathrm{T}, \mathrm{T}^{\prime}$ on $\mathrm{G}$ such that $\mathrm{T} \backslash\{\mathrm{t}\}=\mathrm{T}^{\prime} \backslash\left\{\mathrm{t}^{\prime}\right\}$ for some tubes $t \in T$ and $t^{\prime} \in T^{\prime}$. As mentioned before, the nested complex $\mathcal{N}(G)$ of $G$ can be realized as the boundary complex of a convex polytope [FS05, CD06, Zel06, Pos09, Vol10, DFRS15]. Therefore this complex is an $(n-1)$-dimensional simplicial sphere so that any tube of a maximal tubing can be flipped into another tube, described in the following proposition.

Proposition 4 Let $\mathrm{t}$ be a tube in a maximal tubing $\mathrm{T}$ on $\mathrm{G}$, and let $\overline{\mathrm{t}}$ be the inclusion minimal tube of $\mathrm{T} \cup \kappa(\mathrm{G})$ which strictly contains $\mathrm{t}$. Then the unique tube $\mathrm{t}^{\prime}$ such that $\mathrm{T}^{\prime}=\mathrm{T} \triangle\left\{\mathrm{t}, \mathrm{t}^{\prime}\right\}$ is again a maximal tubing on $\mathrm{G}$ is the connected component of $\mathrm{G}[\overline{\mathrm{t}} \backslash \lambda(\mathrm{t}, \mathrm{T})]$ containing $\lambda(\overline{\mathrm{t}}, \mathrm{T})$.

We say that two distinct tubes $t$ and $t^{\prime}$ of $G$ are exchangeable if there exists two adjacent maximal tubings $T, T^{\prime}$ on $G$ such that $T \backslash\{t\}=T^{\prime} \backslash\left\{t^{\prime}\right\}$. There might be several pairs $\left\{T, T^{\prime}\right\}$ satisfying this condition, but in all cases the tubing $T \cap T^{\prime}$ has to contain some tubes depending only on $t$ and $t^{\prime}$, that we call the forced tubes of the exchangeable pair $\left\{t, t^{\prime}\right\}$. One can observe that the root $\lambda(t, T)$ of $t$ in $T$ and the root $\lambda\left(t^{\prime}, T^{\prime}\right)$ of $t^{\prime}$ in $T^{\prime}$ do not depend on $T$ nor on $T^{\prime}$. The forced tubes are then precisely the tube $\overline{\mathrm{t}}:=\mathrm{t} \cup \mathrm{t}^{\prime}$ and the connected components of $\mathrm{G}\left[\overline{\mathrm{t}} \backslash\left(\lambda(\mathrm{t}, \mathrm{T}) \cup \lambda\left(\mathrm{t}^{\prime}, \mathrm{T}^{\prime}\right)\right)\right]$. Figure 3 gathers illustrations for all notions we just described on a graph $\mathrm{G}_{\mathrm{ex}}$ on 15 vertices.

Now for two tubes $\mathrm{t}, \mathrm{t}^{\prime}$ of $\mathrm{G}$, we define the compatibility degree of $\mathrm{t}$ with $\mathrm{t}^{\prime}$ to be the following quantity:

$$
\left(\mathrm{t} \| \mathrm{t}^{\prime}\right):= \begin{cases}-1 & \text { if } \mathrm{t}=\mathrm{t}^{\prime}, \\ \mid\left\{\text { neighbors of } \mathrm{t} \text { in } \mathrm{t}^{\prime} \backslash \mathrm{t}\right\} \mid & \text { if } \mathrm{t} \not \mathrm{t}^{\prime}, \\ 0 & \text { otherwise }\end{cases}
$$



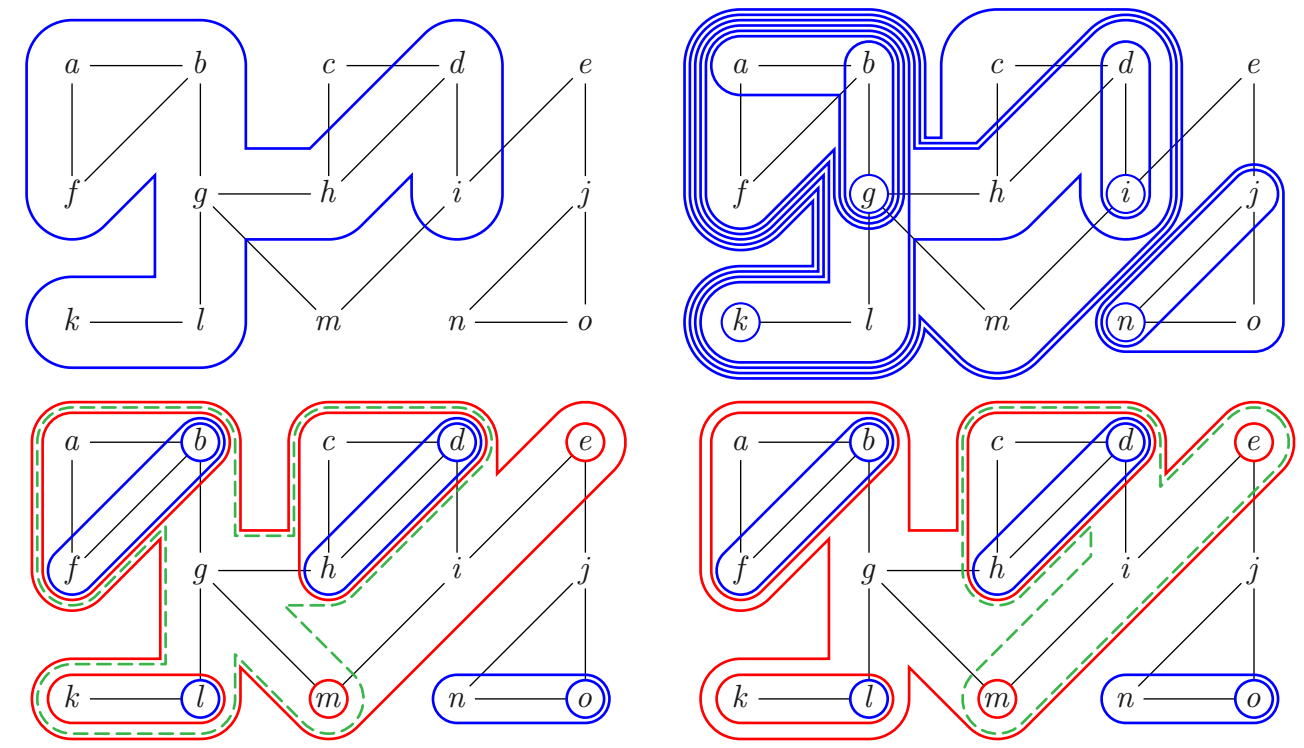

Fig. 3: A tube $\mathrm{t}_{\mathrm{ex}}^{\circ}=\{a, b, d, f, g, h, i, k, l\}$ of $\mathrm{G}_{\mathrm{ex}}$ (top-left), a maximal tubing $\mathrm{T}_{\mathrm{ex}}^{\circ}$ on $\mathrm{G}_{\mathrm{ex}}$ (top-right) and two maximal tubings related by a flip (bottom): the two exchanged tubes $\mathrm{t}_{\mathrm{ex}}=\{a, b, c, d, f, g, h, k, l, m\}$ and $\mathrm{t}_{\mathrm{ex}}^{\prime}=\{c, d, e, h, i, m\}$ are in dotted green while the six forced tubes are in red, and the seven others are in blue.

Example 5 On the graph $\mathrm{G}_{e x}$ of Figure 3 the compatibility degrees of the green tubes $\mathrm{t}_{e x}, \mathrm{t}_{e x}^{\prime}$ (bottom left and right respectively) and of the red tube $\overline{\mathrm{t}}_{e x}=\mathrm{t}_{e x} \cup \mathrm{t}_{e x}^{\prime}$ (bottom) with the tube $\mathrm{t}_{e x}^{\circ}$ (top left) are given by

$$
\begin{aligned}
& \left(\mathrm{t}_{e x} \| \mathrm{t}_{e x}^{\circ}\right)=|\{i\}|=1, \quad\left(\mathrm{t}_{e x}^{\prime} \| \mathrm{t}_{e x}^{\circ}\right)=|\{g\}|=1, \quad\left(\overline{\mathrm{t}}_{e x} \| \mathrm{t}_{e x}^{\circ}\right)=0, \\
& \left(\mathrm{t}_{e x}^{\circ} \| \mathrm{t}_{e x}\right)=|\{c, m\}|=2, \quad\left(\mathrm{t}_{e x}^{\circ} \| \mathrm{t}_{e x}^{\prime}\right)=|\{c, e, m\}|=3, \quad\left(\mathrm{t}_{e x}^{\circ} \| \overline{\mathrm{t}}_{e x}\right)=0 .
\end{aligned}
$$

As the compatibility degree on cluster variables defined in [FZ03], our compatibility degree on tubes encodes compatibility and exchangeability between cluster variables. In particular, it has the following analogous key properties.

Proposition 6 For any two tubes $\mathrm{t}, \mathrm{t}^{\prime}$ of $\mathrm{G}$,

- $\left(\mathrm{t} \| \mathrm{t}^{\prime}\right)$ only depends on $\mathrm{t}$ and $\mathrm{t}^{\prime}$,

- $\left(\mathrm{t} \| \mathrm{t}^{\prime}\right)<0 \Longleftrightarrow\left(\mathrm{t}^{\prime} \| \mathrm{t}\right)<0 \Longleftrightarrow \mathrm{t}=\mathrm{t}^{\prime}$,

- $\left(\mathrm{t} \| \mathrm{t}^{\prime}\right)=0 \Longleftrightarrow\left(\mathrm{t}^{\prime} \| \mathrm{t}\right)=0 \Longleftrightarrow \mathrm{t}$ and $\mathrm{t}^{\prime}$ are compatible,

- $\left(\mathrm{t} \| \mathrm{t}^{\prime}\right)=1=\left(\mathrm{t}^{\prime} \| \mathrm{t}\right) \Longleftrightarrow \mathrm{t}$ and $\mathrm{t}^{\prime}$ are exchangeable.

Fix an initial maximal tubing $\mathrm{T}^{\circ}:=\left\{\mathrm{t}_{1}^{\circ}, \ldots, \mathrm{t}_{n}^{\circ}\right\}$ on $\mathrm{G}$. The primal (resp. dual) compatibility vector of a tube $\mathrm{t}$ with respect to $\mathrm{T}^{\circ}$ is $\mathbf{d}\left(\mathrm{T}^{\circ}, \mathrm{t}\right):=\left[\left(\mathrm{t}_{1}^{\circ} \| \mathrm{t}\right), \ldots,\left(\mathrm{t}_{n}^{\circ} \| \mathrm{t}\right)\right]\left(\operatorname{resp} . \mathbf{d}^{*}\left(\mathrm{t}, \mathrm{T}^{\circ}\right):=\left[\left(\mathrm{t} \| \mathrm{t}_{1}^{\circ}\right), \ldots,\left(\mathrm{t} \| \mathrm{t}_{n}^{\circ}\right)\right]\right)$. The primal (resp. dual) compatibility matrix of a maximal tubing $\mathrm{T}:=\left\{\mathrm{t}_{1}, \ldots, \mathrm{t}_{n}\right\}$ with respect to $\mathrm{T}^{\circ}$ is $\mathbf{d}\left(\mathrm{T}^{\circ}, \mathbf{T}\right):=\left[\left(\mathrm{t}_{i}^{\circ} \| \mathrm{t}_{j}\right)\right]_{i, j \in[n]}\left(\right.$ resp. $\left.\mathbf{d}^{*}\left(\mathrm{~T}, \mathrm{~T}^{\circ}\right):=\left[\left(\mathrm{t}_{i} \| \mathrm{t}_{j}^{\circ}\right)\right]_{i, j \in[n]}\right)$. Our main result is given in the following statement, where $\mathbb{R}_{\geq 0} \mathbf{M}$ denotes the positive span of the column vectors of a matrix $\mathbf{M}$. 

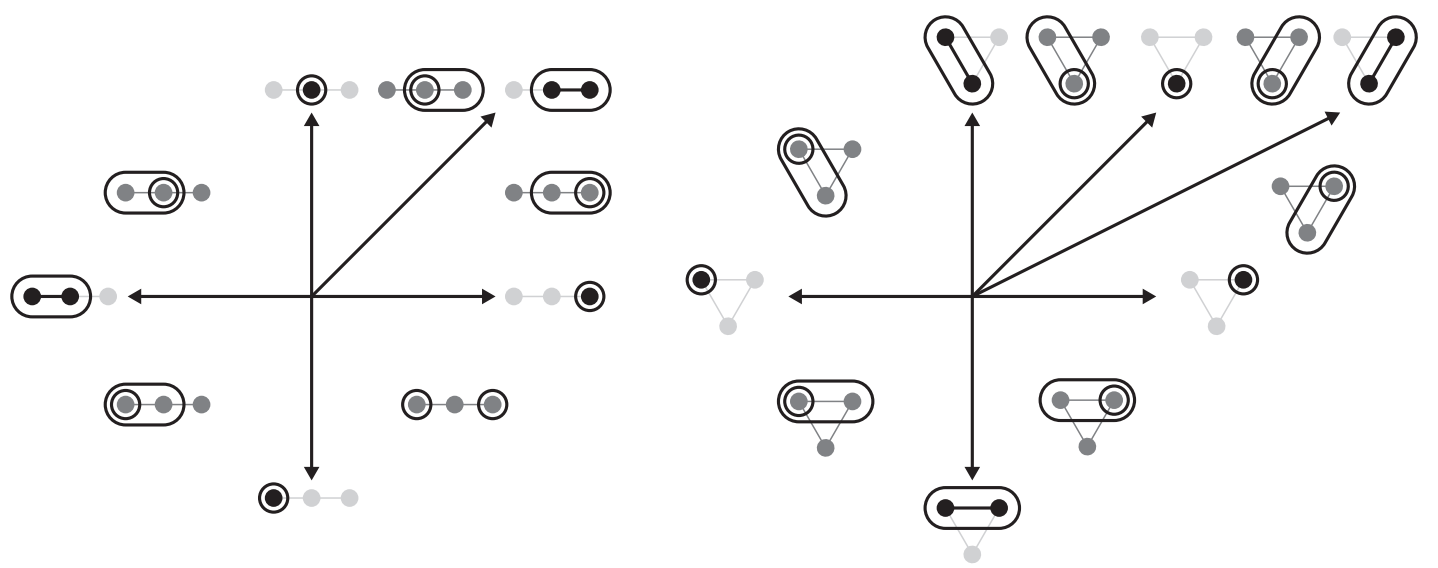

Fig. 4: Compatibility fans of the 3-path (left) and of the triangle (right).

Theorem 7 For any graph G, the compatibility vectors (resp. dual compatibility vectors) of all tubes of G with respect to any initial maximal tubing $\mathrm{T}^{\circ}$ on $\mathrm{G}$ support a complete simplicial fan realizing the nested complex $\mathcal{N}(\mathrm{G})$ on $\mathrm{G}$. More precisely, the two collections of cones,

$\mathcal{D}\left(\mathrm{G}, \mathrm{T}^{\circ}\right):=\left\{\mathbb{R}_{\geq 0} \mathbf{d}\left(\mathrm{T}^{\circ}, \mathrm{T}\right) \mid \mathrm{T}\right.$ tubing on $\left.\mathrm{G}\right\} \quad$ and $\quad \mathcal{D}^{*}\left(\mathrm{G}, \mathrm{T}^{\circ}\right):=\left\{\mathbb{R}_{\geq 0} \mathbf{d}^{*}\left(\mathrm{~T}, \mathrm{~T}^{\circ}\right) \mid \mathrm{T}\right.$ tubing on $\left.\mathrm{G}\right\}$

are complete simplicial fans. We respectively call them the compatibility fan and the dual compatibility fan of $\mathrm{G}$ with respect to $\mathrm{T}^{\circ}$.

Figure 4 shows the compatibility fans of the 3-path and of the triangle (only connected graphs with 3 vertices). Any other choice of initial tubing would produce the same fans: it is clear for the triangle as all maximal tubings are equivalent under graph isomorphisms and it is easy to check for the path. The first interesting compatibility fans appear in dimension 3 for connected graphs on 4 vertices. All possibilities up to linear transformations are represented in Figure 5 for the path, the cycle, the complete graph and the star on 4 vertices. All other compatibility fans on connected graphs with 4 vertices can be found in [MP15]. Instead of representing cones in the 3-dimensional space, we intersect the compatibility vectors with the unit sphere, make a stereographic projection of the resulting points on the sphere (the pole of the projection is the point of the sphere in direction $-\mathbf{e}_{1}-\mathbf{e}_{2}-\mathbf{e}_{3}$ ), and draw the cones on the resulting planar points. Under this projection, the three external vertices correspond to the tubes of the initial tubing, and the external face corresponds to the initial tubing. For the sake of readability, we only gave the labels of the remaining vertices of the top pictures of Figure 5 while we only labeled the initial tubes for the others. All labels can indeed be reconstructed from the initial tubes by flips. The pictures become more complicated in dimension 4. Figure 6 represents the stereographic projection of one compatibility fan for the path, the cycle, the complete graph, and the star on 5 vertices.

The next step would be to find convex polytopes with our fans as normal fans. This question remains open for now, except for some particular graphs. We checked it computationally for all graphs with at most 4 vertices, for complete graphs with at most 7 vertices, and we settled the case of paths and cycle in Theorem 2 which follows a similar proof as [CSZ15]. We conjecture a positive answer in full generality.

Conjecture 8 All primal and dual compatibility fans of any graph $\mathrm{G}$ are normal fans of convex polytopes. 

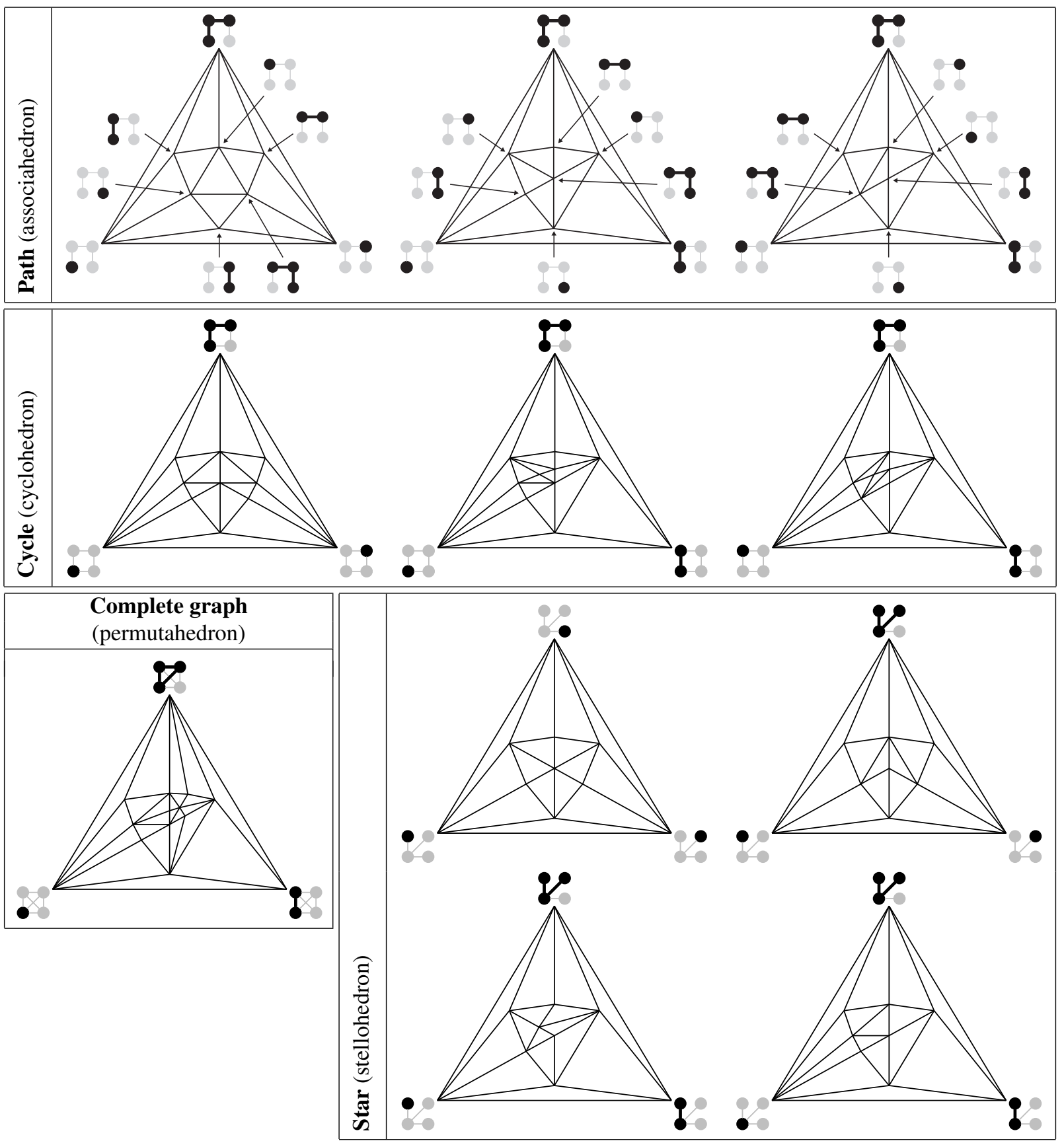

Fig. 5: All possible compatibility fans up to linear isomorphism, for the path, the cycle, the complete graph and the star on 4 vertices. Instead of representing the cones in the 3 -dimensional space, we intersect the compatibility vectors with the unit sphere, make a stereographic projection of the resulting points on the sphere (the pole of the projection is the point of the sphere in direction $-\mathbf{e}_{1}-\mathbf{e}_{2}-\mathbf{e}_{3}$ ), and draw the cones on the resulting points in the plane. 

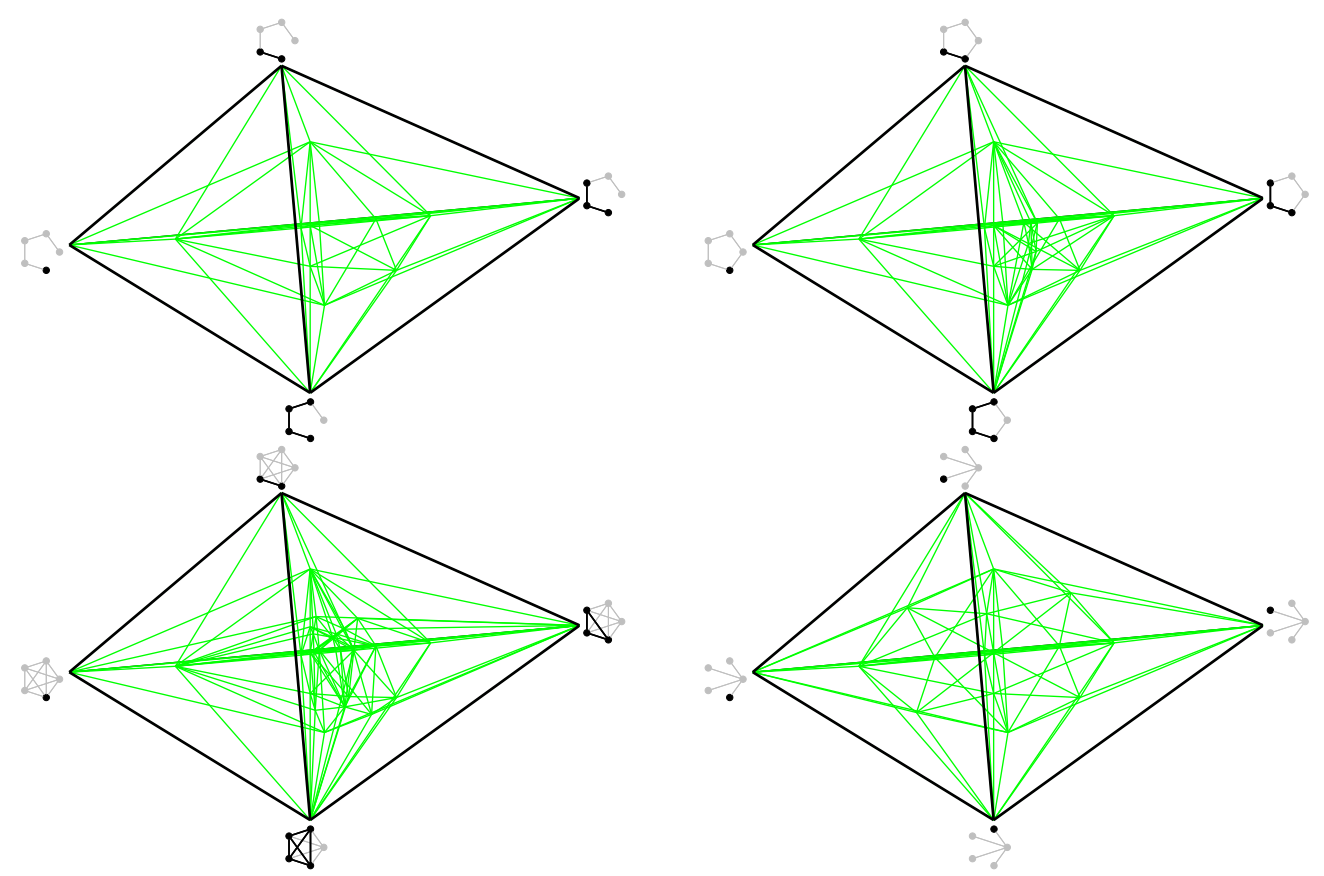

Fig. 6: Stereographic projection of one compatibility fan for the path, cycle, complete graph and star on 5 vertices.

\section{Link with type $A, B$ and $C$ cluster complexes}

We now show that our compatibility degree in path and cycle nested complexes matches the compatibility degree in the type $A, B$ and $C$ cluster complexes of S. Fomin and A. Zelevinsky [FZ03, CP15].

\subsection{Type $A$ and paths}

The $n$-dimensional cluster complex of type $A$ is the classical simplicial associahedron. Its combinatorial model is the simplicial complex of sets of pairwise noncrossing diagonals of an $(n+3)$-gon. It can be seen as the nested complex of an $(n+1)$-path $\mathrm{P}_{n+1}$ : consider an $(n+3)$-gon $Q_{n+3}$ with vertices labeled from left to right by $0,1, \ldots, n+2$ and such that $1, \ldots, n+1$ are located below the edge $[0, n+2]$ (see Figure 7). We identify $\mathrm{P}_{n+1}$ with the path $1, \ldots, n+1$ on the boundary of $Q_{n+3}$, and associate to a diagonal $\delta$ of $Q_{n+3}$ the tube $\mathrm{t}_{\delta}$ of $\mathrm{P}_{n+1}$ whose vertices are located strictly below $\delta$ (see Figure 7). We associate to a set $\Delta$ of pairwise noncrossing internal diagonals of $Q_{n+3}$ the set of tubes $\mathrm{T}_{\Delta}:=\left\{\mathrm{t}_{\delta} \mid \delta \in \Delta\right\}$. The map $\Delta \mapsto \mathrm{T}_{\Delta}$ is an isomorphism between the simplicial associahedron and the nested complex $\mathcal{N}\left(\mathrm{P}_{n+1}\right)$. The behavior of this isomorphism with respect to the compatibility degrees is described as follows.

Proposition 9 For any two internal diagonals $\delta$ and $\delta^{\prime}$ of $Q_{n+3}$, the compatibility degree between the corresponding tubes $\mathrm{t}_{\delta}$ and $\mathrm{t}_{\delta^{\prime}}$ of $\mathrm{P}_{n+1}$ is given by the following formula.

$$
\left(\mathrm{t}_{\delta} \| \mathrm{t}_{\delta^{\prime}}\right)= \begin{cases}-1 & \text { if } \delta=\delta^{\prime} \\ 0 & \text { if } \delta \neq \delta^{\prime} \text { do not cross } \\ 1 & \text { if } \delta \neq \delta^{\prime} \text { cross } .\end{cases}
$$



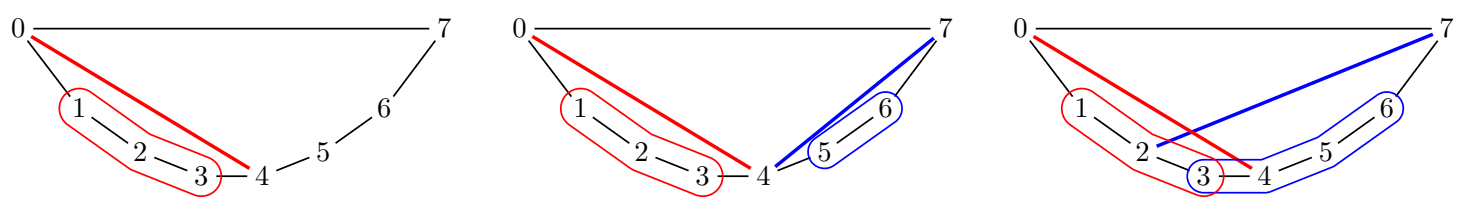

$$
1-2-3-4-5-6
$$

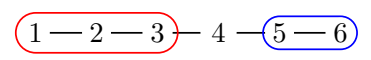

$1-2-3-4-5-6$

Fig. 7: Isomorphism between the simplicial associahedron and the nested complex of a path: it sends the diagonals of the polygon to tubes (left), and preserves both compatibility (middle) and incompatibility (right).

In other words, our compatibility degree between tubes of $\mathrm{P}_{n+1}$ coincides with the compatibility degree of S. Fomin and A. Zelevinsky in [FZ03] between type $A$ cluster variables. Our compatibility fans for paths are thus type $A$ denominator fans, and we obtain a new proof of F. Santos' result [CSZ15, Section 5].

\subsection{Types $B$ and $C$, and cycles}

The $n$-dimensional cluster complex of type $B$ is the classical simplicial cyclohedron. Its combinatorial model is the simplicial complex of sets of pairwise noncrossing pairs of centrally symmetric diagonals (and duplicated long diagonals) of a regular $(2 n+2)$-gon $R_{2 n+2}$. It can be seen as the nested complex of an $(n+1)$-cycle $\mathrm{O}_{n+1}$ : we label the vertices of $R_{2 n+2}$ cyclically with two copies of $[n+1]$ and associate

- to a duplicated long diagonal $\delta$ with vertices labeled by $i$ the tube $\mathrm{t}_{\delta}:=[n+1] \backslash\{i\}$ of $\mathrm{O}_{n+1}$,

- to a pair of centrally symmetric diagonals $\{\delta, \bar{\delta}\}$ the tube $\mathrm{t}_{\delta}$ of $\mathrm{O}_{n+1}$ which consists in the labels of the vertices of $R_{2 n+2}$ that are strictly separated from the center of $R_{2 n+2}$ by $\delta$ and $\bar{\delta}$ (see Figure 8 .

As we did in type $A$, we finally associate to a set $\Delta$ of pairwise noncrossing pairs of centrally symmetric diagonals of $R_{2 n+2}$ the set of tubes $\mathrm{T}_{\Delta}:=\left\{\mathrm{t}_{\delta} \mid \delta \in \Delta\right\}$. The map $\Delta \mapsto \mathrm{T}_{\Delta}$ defines an isomorphism between the simplicial cyclohedron and the nested complex $\mathcal{N}\left(\mathrm{O}_{n+1}\right)$. This isomorphism behaves as follows with respect to the compatibility degrees.

Proposition 10 For any two pairs of centrally symmetric diagonals (or duplicated long diagonals) $\{\delta, \bar{\delta}\}$ and $\left\{\delta^{\prime}, \bar{\delta}^{\prime}\right\}$ of the $(2 n+2)$-gon $R_{2 n+2}$, the compatibility degree $\left(\mathrm{t}_{\delta} \| \mathrm{t}_{\delta^{\prime}}\right)$ of the corresponding tubes $\mathrm{t}_{\delta}$ and $\mathrm{t}_{\delta^{\prime}}$ of $\mathrm{O}_{n+1}$ is the number of crossings between the two diagonals $\delta$ and $\bar{\delta}$ and the diagonal $\delta^{\prime}$.

Our compatibility (resp. dual compatibility) degree between tubes of $\mathrm{O}_{n+1}$ thus coincides with the compatibility degree of S. Fomin and A. Zelevinsky in [FZ03] between type $C$ (resp. $B$ ) cluster variables. Our graphical compatibility (resp. dual compatibility) fans for cycles are thus type $C$ (resp. $B$ ) denominator fans.

Finally, as we have a good control on the geometry of the compatibility fans for paths and cycles (in particular, a sufficient understanding of the linear dependencies among the compatibility vectors of tubes involved in a flip), we can give explicit height functions showing that all the paths and cycles compatibility fans are polytopal. The following statement summarizes the results of this section.

Theorem 11 In types $A, B$ and $C$, the denominator vectors (or compatibility vectors) of cluster variables with respect to any initial cluster support a complete simplicial fan realizing the cluster complex. Moreover, this fan is the normal fan of a simple convex polytope. 

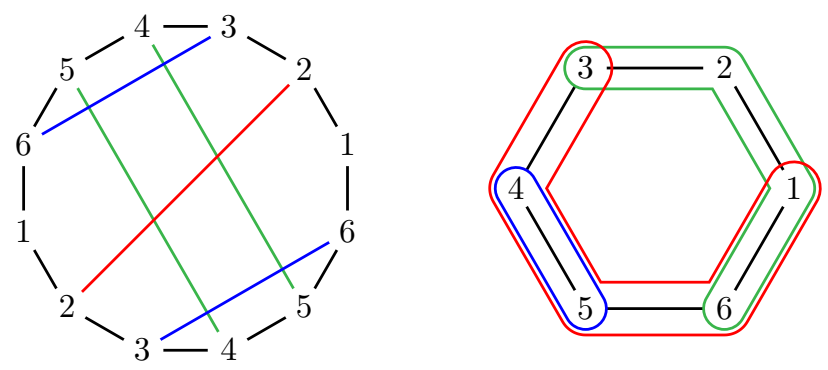

Fig. 8: Isomorphism between the simplicial cyclohedron and the nested complex of a cycle: it sends centrally symmetric pairs of diagonals of the polygon (left) to tubes (right), and preserves both compatibility and incompatibility.

\section{A sketch of proof for the main statement}

Our proof for Theorem 7 relies on the following classical result of polyhedral geometry. We follow the presentation of [DRS10, Corollary 4.5.20].

Proposition 12 For a simplicial sphere $\Delta$ with vertex set $X$ and a set of vectors $\mathbf{V}:=\left(\mathbf{v}_{x}\right)_{x \in X}$ of $\mathbb{R}^{n}$, the collection of cones $\left\{\mathbb{R}_{\geq 0} \mathbf{V}_{\triangle} \mid \Delta \in \Delta\right\}$, where $\mathbb{R}_{\geq 0} \mathbf{V}_{\triangle}$ denotes the positive span of the set of vectors $\mathbf{V}_{\triangle}:=\left\{\mathbf{v}_{x} \mid x \in \triangle\right\}$, forms a complete simplicial fan if and only if

1. there exists a facet $\triangle$ of $\Delta$ such that $\mathbf{V}_{\triangle}$ is a basis of $\mathbb{R}^{n}$ and such that the open cones $\mathbb{R}_{>0} \mathbf{V}_{\triangle}$ and $\mathbb{R}_{>0} \mathbf{V}_{\triangle^{\prime}}$ have an empty intersection for any facet $\triangle^{\prime}$ of $\Delta$ distinct from $\triangle$;

2. for any two adjacent facets $\triangle, \Delta^{\prime}$ of $\Delta$ with $\triangle \backslash\{x\}=\Delta^{\prime} \backslash\left\{x^{\prime}\right\}$, the coefficients $\alpha, \alpha^{\prime}$ have the same sign (and different from 0 ) in the unique (up to rescaling) linear dependence on $\mathbf{V}_{\triangle \cup \triangle^{\prime}}$

$$
\alpha \mathbf{v}_{x}+\alpha^{\prime} \mathbf{v}_{x^{\prime}}+\sum_{y \in \Delta \cap \triangle^{\prime}} \beta_{y} \mathbf{v}_{y}=0 .
$$

The properties of our degree (see Proposition 6 imply that the initial tubing $\mathrm{T}^{\circ}:=\left\{\mathrm{t}_{1}^{\circ}, \ldots, \mathrm{t}_{n}^{\circ}\right\}$ satisfies the first condition of Proposition 12. So we just need to check the second condition, which in our setting rephrases as follows. For any two adjacent maximal tubings $T, T^{\prime}$ on $G$, with $T \backslash\{t\}=T^{\prime} \backslash\left\{t^{\prime}\right\}$, the coefficients of $\mathbf{d}\left(T^{\circ}, t\right)$ and $\mathbf{d}\left(T^{\circ}, t^{\prime}\right)$ (resp. of $\mathbf{d}^{*}\left(t, T^{\circ}\right)$ and $\mathbf{d}^{*}\left(t^{\prime}, T^{\circ}\right)$ ) in the linear dependence on the primal (resp. dual) compatibility vectors of the tubes of $T \cup \mathrm{T}^{\prime}$ have the same sign.

A first trick allows us to restrict the proof only to primal compatibility fans: the previous condition can be reformulated as a constraint on the determinants of the compatibility matrices of $T$ and $\mathrm{T}^{\prime}$, namely $\operatorname{det}\left(\mathbf{d}\left(\mathbf{T}^{\circ}, \mathbf{T}\right)\right) \cdot \operatorname{det}\left(\mathbf{d}\left(\mathbf{T}^{\circ}, \mathbf{T}^{\prime}\right)\right)<0$. Using the straightforward equality $\mathbf{d}^{*}\left(\mathbf{T}, \mathbf{T}^{\circ}\right)=\mathbf{d}\left(\mathbf{T}, \mathbf{T}^{\circ}\right)^{t}$, we obtain that the products $\operatorname{det}\left(\mathbf{d}^{*}\left(T, T^{\circ}\right)\right) \cdot \operatorname{det}\left(\mathbf{d}^{*}\left(\mathbf{T}^{\prime}, T^{\circ}\right)\right)$ have the same signs for all initial maximal tubings $\mathbf{T}^{\circ}$. This concludes since $\operatorname{det}\left(\mathbf{d}^{*}(\mathbf{T}, \mathbf{T})\right) \cdot \operatorname{det}\left(\mathbf{d}^{*}\left(\mathbf{T}^{\prime}, \mathbf{T}\right)\right)<0$ by Proposition 6 .

Consider now two adjacent maximal tubings $T, T^{\prime}$ with $T \backslash\{t\}=T^{\prime} \backslash\left\{t^{\prime}\right\}$. As mentioned in Section 1 . the tubings $T$ and $T^{\prime}$ both have to contain the tube $\overline{\mathrm{t}}:=\mathrm{t} \cup \mathrm{t}^{\prime}$ and the connected components $\underline{\mathrm{t}}_{1}, \ldots \underline{\mathrm{t}}_{k}$ of $G\left[t \cap t^{\prime}\right]$. Suppose that an initial tube $t_{i}^{\circ}$ is not contained in $\bar{t}$, then its degree with any tube $t^{\prime \prime}$ contained in $\overline{\mathrm{t}}$ is given by $\left(\mathrm{t}_{i}^{\circ} \| \mathrm{t}^{\prime \prime}\right)=\mid\left\{\right.$ neighbors of $\mathrm{t}_{i}^{\circ}$ in $\left.\mathrm{t}^{\prime \prime} \backslash \mathrm{t}_{i}^{\circ}\right\} \mid$. Writing $\left(\mathrm{t}_{i}^{\circ} \| \underline{\mathrm{t}}\right)$ for $\sum_{1 \leq \ell \leq k}\left(\mathrm{t}_{i}^{\circ} \| \underline{\mathrm{t}}_{\ell}\right)$, we derive by inclusion-exclusion that $\left(\mathrm{t}_{i}^{\circ} \| \mathrm{t}\right)+\left(\mathrm{t}_{i}^{\circ} \| \mathrm{t}^{\prime}\right)=\left(\mathrm{t}_{i}^{\circ} \| \overline{\mathrm{t}}\right)+\left(\mathrm{t}_{i}^{\circ} \| \underline{\mathrm{t}}\right)$.

If now no initial tube is contained in $\overline{\mathrm{t}}$, then this formula is fulfilled coordinatewise by the compatibility vectors of $\mathrm{t}, \mathrm{t}^{\prime}, \overline{\mathrm{t}}$ and the components of $\mathrm{t}$. So it is the linear dependence that we are looking for, and it 
has the property we expect. The problem is that the compatibility degree sometimes equals zero without reflecting any cardinality. In this case it means that an initial tube is contained in $\bar{t}$. We solve this problem by showing something more precise, and thus stronger, than the second condition of Proposition 12 .

Theorem 13 Let $\mathrm{G}$ be a simple graph and $\mathrm{T}^{\circ}$ an initial maximal tubing on $\mathrm{G}$. Let $\mathrm{T}, \mathrm{T}^{\prime}$ be two adjacent maximal tubings on $\mathrm{G}$ with $\mathrm{T} \backslash\{\mathrm{t}\}=\mathrm{T}^{\prime} \backslash\left\{\mathrm{t}^{\prime}\right\}$. In the linear dependence on compatibility vectors of the tubes in $\mathrm{T} \cup \mathrm{T}^{\prime}$ with respect to $\mathrm{T}^{\circ}$, the coefficients of $\mathrm{d}\left(\mathrm{T}^{\circ}, \mathrm{t}\right)$ and $\mathrm{d}\left(\mathrm{T}^{\circ}, \mathrm{t}^{\prime}\right)$ have the same sign, and the support of the dependence is contained in compatibility vectors of tubes of $\mathrm{T} \cup \mathrm{T}^{\prime}$ contained in $\overline{\mathrm{t}}:=\mathrm{t} \cup \mathrm{t}^{\prime}$.

Theorem 13 is obtained by induction on the size of $\bar{t}$. To do this, we need additional technical results, in particular a method to find a first suitable linear dependence for a pair of exchangeable tubes $t$ and $t^{\prime}$ of G. For this we suppose that the previous inclusion-exclusion formula do not hold, so that some initial tubes are contained in $\bar{t}$. We then define adjacent maximal tubings $\mathrm{T}, \mathrm{T}^{\prime}$ on $\mathrm{G}$ with $\mathrm{T} \backslash\{\mathrm{t}\}=\mathrm{T}^{\prime} \backslash\left\{\mathrm{t}^{\prime}\right\}$ in which some tubes contained in $\bar{t}$ control the contribution of such initial tubes. See [MP15] for details.

\section{Many nonequivalent fan realizations}

We conclude this extended abstract with some considerations on the number of distinct compatibility fans obtained in Theorem 7] up to geometrical equivalence. Following [CSZ15], we consider that two compatibility fans of two graphs $G$ and $G^{\prime}$ are equivalent if they differ by a linear isomorphism. Such a linear isomorphism induces an isomorphism between the nested complexes $\mathcal{N}(\mathrm{G})$ and $\mathcal{N}\left(\mathrm{G}^{\prime}\right)$, which is in turn induced by a graph isomorphism between $\mathrm{G}$ and $\mathrm{G}^{\prime}$, except maybe for graphs that we call spiders (consisting of disjoint paths one of whose endpoints belong to a single clique, see Figure 9).

Theorem 14 The nested complex isomorphisms $\mathcal{N}(\mathrm{G}) \rightarrow \mathcal{N}\left(\mathrm{G}^{\prime}\right)$ are exactly those induced by graph isomorphisms $\mathrm{G} \rightarrow \mathrm{G}^{\prime}$, unless $\mathrm{G}$ and $\mathrm{G}^{\prime}$ are isomorphic and have a connected component being a spider.

Corollary 15 If no connected component of $\mathrm{G}$ is a spider, then equivalence classes of compatibility fans of $\mathrm{G}$ are in bijection with orbits of the action of the automorphism group of $\mathrm{G}$ on maximal tubings on $\mathrm{G}$.

When $\mathrm{G}$ is a spider, the automorphism group of $\mathcal{N}(\mathrm{G})$ is generated by automorphisms induced by graph isomorphisms together with an additional nested complex automorphism $\Omega$ with the property that $\left(t \| t^{\prime}\right)=\left(\Omega\left(t^{\prime}\right) \| \Omega(t)\right)$ for any two tubes $t, t^{\prime}$ of $G$. The action of $\Omega$ on tubes is illustrated in the example of Figure 9 Details can be found in [MP15].
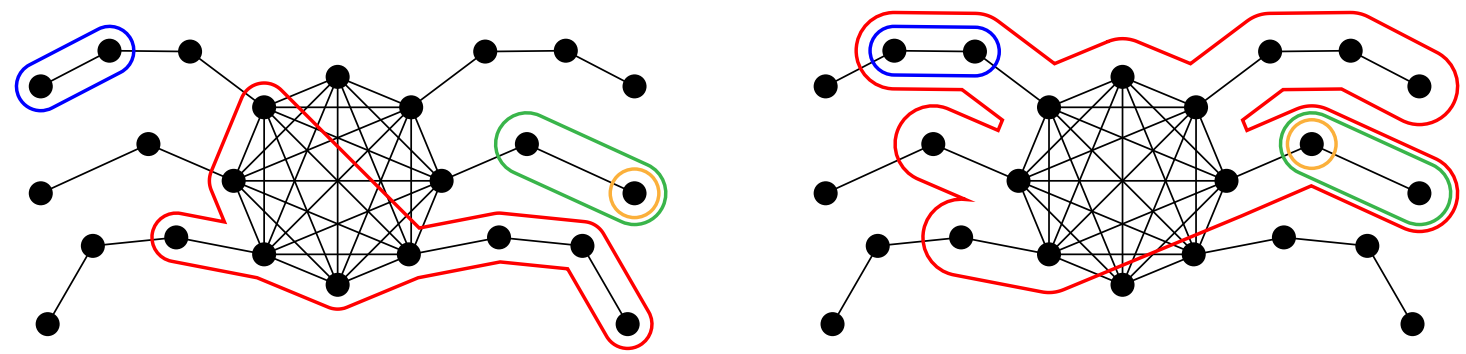

Fig. 9: A spider with a tubing $\mathrm{T}$ (left) and its image $\Omega(\mathrm{T})$ (right) by the nested complex automorphism $\Omega$ of the spider. 


\section{References}

[CD06] M. Carr and S. Devadoss. Coxeter complexes and graph-associahedra. Topology Appl., 153(12):2155-2168, 2006.

[CFZ02] F. Chapoton, S. Fomin, and A. Zelevinsky. Polytopal realizations of generalized associahedra. Canad. Math. Bull., 45(4):537-566, 2002.

[CP15] C. Ceballos and V. Pilaud. Denominator vectors and compatibility degrees in cluster algebras of finite types. Trans. Amer. Math. Soc., 367:1421-1439, 2015.

[CSZ15] C. Ceballos, F. Santos, and G. M. Ziegler. Many non-equivalent realizations of the associahedron. Combinatorica, 35(5):513-551, 2015.

[DFRS15] S. Devadoss, S. Forcey, S. Reisdorf, and P. Showers. Convex polytopes from nested posets. European J. Combin., 43:229-248, 2015.

[DRS10] J. De Loera, J. Rambau, and F. Santos. Triangulations: Structures for Algorithms and Applications, volume 25 of Algorithms and Computation in Mathematics. Springer Verlag, 2010.

[FS05] E. M. Feichtner and B. Sturmfels. Matroid polytopes, nested sets and Bergman fans. Port. Math. (N.S.), 62(4):437-468, 2005.

[FZ02] S. Fomin and A. Zelevinsky. Cluster algebras. I. Foundations. J. Amer. Math. Soc., 15(2):497529, 2002.

[FZ03] S. Fomin and A. Zelevinsky. Cluster algebras. II. Finite type classification. Invent. Math., 154(1):63-121, 2003.

[HL07] C. Hohlweg and C. Lange. Realizations of the associahedron and cyclohedron. Discrete Comput. Geom., 37(4):517-543, 2007.

[Lee89] C. Lee. The associahedron and triangulations of the $n$-gon. European J. Combin., 10(6):551560, 1989.

[Lod04] J.-L. Loday. Realization of the Stasheff polytope. Arch. Math. (Basel), 83(3):267-278, 2004.

[MP15] T. Manneville and V. Pilaud. Compatibility fans for graphical nested complexes. Preprint, arXiv:1501.07152, 2015.

[Pos09] A. Postnikov. Permutohedra, associahedra, and beyond. Int. Math. Res. Not. IMRN, (6):1026$1106,2009$.

[Ste13] S. Stella. Polyhedral models for generalized associahedra via Coxeter elements. J. Algebraic Combin., 38(1):121-158, 2013.

[Vol10] V. D. Volodin. Cubical realizations of flag nestohedra and a proof of Gal's conjecture for them. Uspekhi Mat. Nauk, 65(1(391)):183-184, 2010.

[Zel06] A. Zelevinsky. Nested complexes and their polyhedral realizations. Pure Appl. Math. Q., 2(3):655-671, 2006. 